\title{
Submitted: The usefulness of high-frequency ultrasonography Accepted: 20.11.2019 in the evaluation of vulvar dermatoses in postmenopausal women - a preliminary report
}

Published:

31.12.2019

Keywords

high-frequency ultrasonography, vulvar dermatoses, ultrasound, vulva

\author{
Michał Migda1,2, Marian Stanisław Migda², Bartosz Migda³, \\ Marek Maleńczyk ${ }^{1}$
}

${ }^{1}$ Clinical Unit of Obstetrics, Women's Disease and Gynecological Oncology, United District Hospital, Collegium Medicum University of Nicolaus Copernicus in Toruń, Torun, Poland

${ }^{2}$ Civis Vita Medical Center Torun, Poland

${ }^{3}$ Department of Diagnostic Imaging, Second Faculty of Medicine with the English Division and the Physiotherapy Division, Medical University of Warsaw, Warsaw, Poland

Correspondence: Michat Migda, Warszawska 20, 87-100 Toruń; tel.: 5664476 07, e-mail:mchmigda@gmail.com

DOI: $10.15557 / \mathrm{JoU} .2019 .0042$

\begin{abstract}
Introduction: The vulva is an external female genital organ with complex anatomy and histology. In postmenopausal women, this region is at particular risk of different types of conditions known as dermatoses. Materials and methods: We assessed benign vulvar skin lesions using high-frequency $48 \mathrm{MHz}$ ultrasound transducer (DermaView) prior to biopsy or excision. We compared ultrasonographic images with histology. Results: We assessed benign vulvar lesions such as folliculitis, condylomata acuminatum, lichen sclerosus and vulvar intraepithelial neoplasia. We presented typical high-frequency images of these pathologies and compared them with histological images. Conclusion: Our preliminary study confirms that high-frequency ultrasonography is a useful tool in the assessment of vulvar pathologies, especially before surgical excision. A precise visualization of certain pathologies like folliculitis, lichen sclerosus, condylomata and vulvar intraepithelial neoplasia is possible.
\end{abstract}

\section{Introduction}

The vulva is an external female genital organ, which has a complex anatomy composed of hymen, anal margin, labia minora, labia majora and clitorial hood ${ }^{(1)}$. Different types of epithelium predispose to a wide range of conditions known as vulvar dermatoses ${ }^{(2,3)}$. Vulvovaginal tissues are particularly susceptible to many irritants and allergens compared to other skin $\operatorname{sites}^{(4)}$. Tissue atrophy and immunologic changes that occur with aging increase the risk of the most common vulvar dermatoses ${ }^{(2)}$, such as inflammatory disorders ${ }^{(3)}$, infections (folliculitis, condyloma accuminata) and precancerous conditions (vulvar intraepithelial neoplasia and vulvar Paget's disease $)^{(5)}$. Due to the unique location and anatomy, understanding VD requires knowledge in both dermatopathology and gynecologic pathology ${ }^{(6)}$. Nowadays, vulvar diagnosis is based on macroscopic evaluation, physical examination, vulvoscopy, microbiological specimen collection and biopsy ${ }^{(7)}$. Recent publications highlight the utility of high-frequency $40-50 \mathrm{MHz}$ transducers in detailed imaging of epidermal echo, dermis, and the upper part of subcutaneous tissue, skin appendages (hair, follicles) as well as vessels running in the dermis and upper subcutaneous tissue, and pathologically altered $\operatorname{skin}^{(7,8)}$ In this study, we analyzed clinical, ultrasonographic and histological images to correlate HFUS with histological images. 


\section{Materials and methods}

The study was approved by the board of Clinical Unit of Obstetrics, Women's Disease and Gynecological Oncology, United District Hospital, Collegium Medicum University of Nicolaus Copernicus in Torun, Poland. Four cases of postmenopausal women referred to our clinic for biopsy were included in the analysis. In this study, we performed high-frequency ultrasonography (HFUS) using $48 \mathrm{MHz}$ DermaView mechanical probe scanner (Dramiński S.A., Poland) prior to surgical excision. The collected specimens of altered tissue were formalin-fixed and paraffin-embedded. The paraffin-embedded blocks were cut into $4 \mu \mathrm{m} \mathrm{sec-}$ tions for a routine histology evaluation.

\section{Results}

\section{Folliculitis}

Folliculitis is a common skin condition in which a hair follicle becomes inflamed. It is caused by bacterial or fungal infection. Most of cases resolve spontaneously within a few days. In the case of our patient, the lesion was not resolving for 2 months despite antibiotic and steroid treatment. The patient was qualified for histological verification (Fig. 1). HFUS showed a well-defined hypoechoic subepidermal lesion as compared to adjacent tissue, with sharp margin, without posterior acoustic enhancement extending to the dermis (Fig. 2). A histological evaluation was performed to to verify ultrasonographic findings (Fig. 3).

\section{Condyloma accuminatum}

Condyloma (from Greek "knuckle") refers to papular genital wart-like growths. Condyloma acuminatum refers to single lesions. Genital warts are a productive vegetative manifestation of epithelial infection caused by human papillomavirus (HPV), which infects epithelial basal cells. Although 90\% of those who contact HPV will not develop warts, they can still transmit the virus ${ }^{(9)}$. However, approximately $10 \%$ of individuals develop a persistent infection, with a risk of developing benign proliferative lesions, high-grade precursors or eventually invasive carcinoma ${ }^{(10)}$. Complete surgical excision with histologically clear margins in cases of single lesion is the best treatment approach ${ }^{(9)}$. A 70-year-old woman with condyloma acuminatum was referred to our clinic for histological evaluation (Fig. 4). HFUS was performed prior to surgery. Condyloma presented in HFUS as a hypoechoic homogeneous lesion compared to the adjacent tissue, with sharp margin, located within the epidermis and dermis, without subcutaneous tissue involvement (Fig. 5). Final histology confirmed condyloma acuminatum with a clear margin of excised lesion (Fig. 6).

\section{Lichen sclerosus}

Lichen sclerosus (LS) is a chronic inflammatory disease with unknown pathophysiology. Potential factors

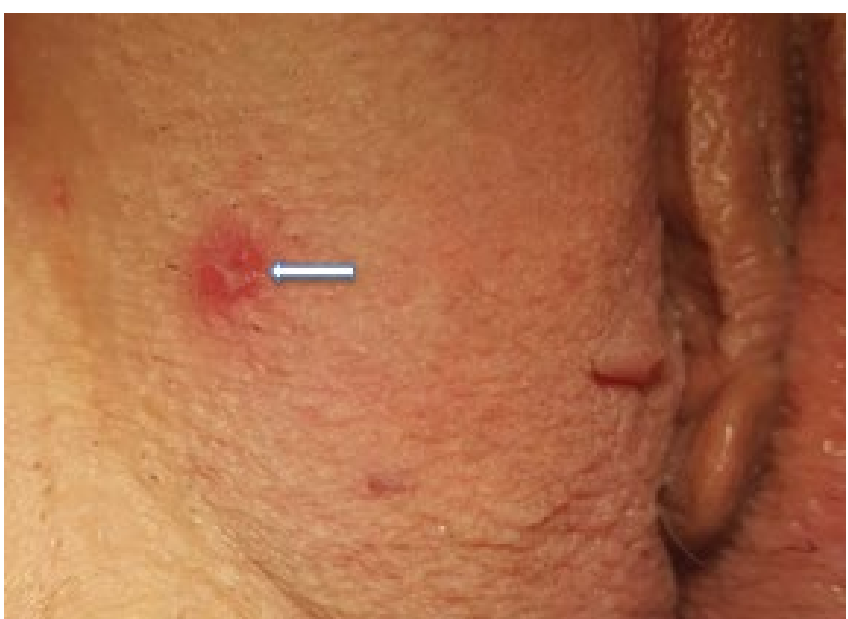

Fig. 1. Macroscopic image of folliculitis, skin inflammation (arrow)

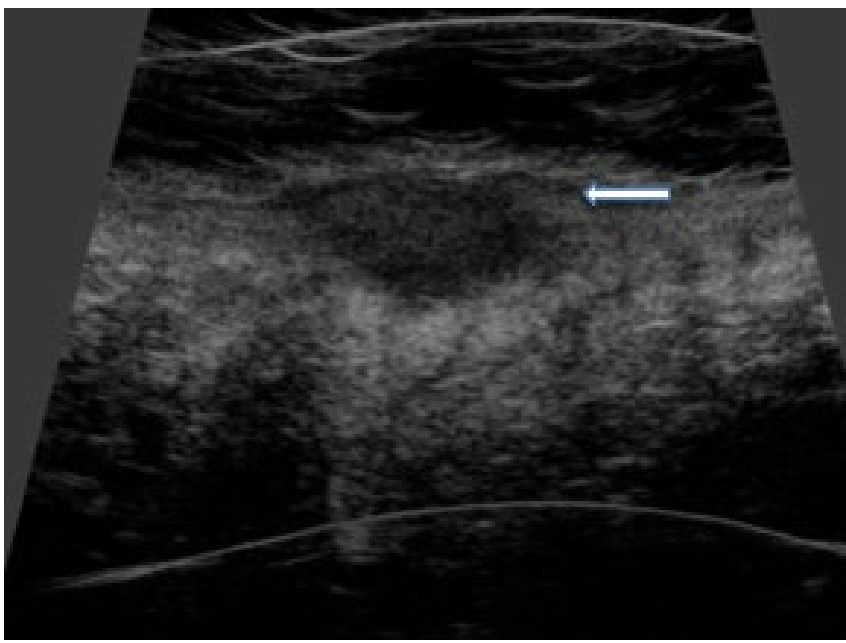

Fig. 2. Folliculitis. HFUS (48 MHz) showing a well-defined nodular hypoechoic mass in epidermis and extending to the subepidermal region. Thin subepidermal low-echogenicity band is present (arrow)

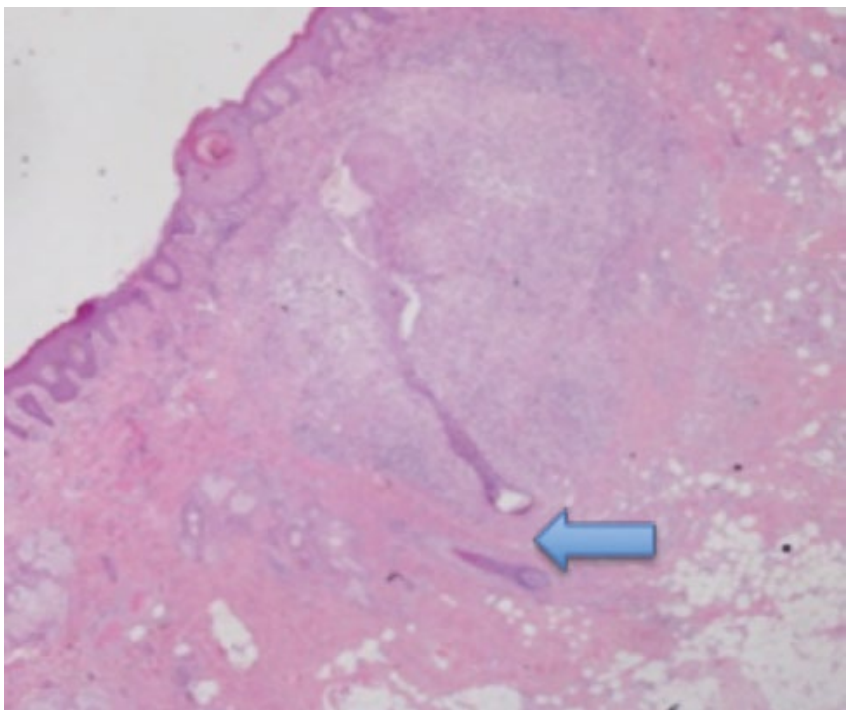

Fig. 1. Follicutis. Histology: accumulation of lymphocytes and other inflammatory cells around a hair follicle ( $H$ and $E)$ 
include genetic predisposition, autoimmune disorders, local immune response, sex hormone factors (low estrogen levels) and some infections ${ }^{(11,12)}$. Although the disease may occur at any age, there is a typical bimodal onset in prepubertal girls and peri- or postmenopausal women ${ }^{(12)}$. Malignant transformation to squamous cell carcinoma has been reported in $2-5 \%$ of patients ${ }^{(11)}$. Differential diagnosis includes lichen planus, psoriasis, lichen simplex chronicus, vitiligo, immunobullous disease and mucous membrane pemphigoid ${ }^{(11,12)}$. Our patient was an 80 -year-old woman suffering from intractable pruritus worsening at night, pruritus ani, irritation and soreness (Fig. 7). The characteristic ultrasound features were hyperechoic entry echo, and thin dermis with typical hypoechoic upper part of dermis. The border between dermis and subcutaneous tissue was linear (Fig. 8). Histological examination confirmed lichen sclerosus of vulva (Fig. 9).

\section{Vulvar intraepithelial neoplasia}

The incidence of vulvar intraepithelial neoplasia (VIN) has increased over years to 2.86 per 100,000 population in $2000^{(13,14)}$. This is associated with increased incidence of HPV infections ${ }^{(13)}$. Originally, VIN lesions were classified as VIN 1-3. In 2004, the International Society for the Study of Vulvovaginal Diseases (ISSVD) developed new classification for VIN. Usual VIN (uVIN) is strongly associated with HPV infection. Differentiated VIN (dVIN) is associated with lichen sclerosus ${ }^{(15)}$. The dVIN is more common in older women and represents only about $5 \%$ of VIN lesions. One systematic review reported progression of VIN 3 to invasive disease in $9 \%$ of 88 patients within a period of 1 to 8 years ${ }^{(16)}$. Despite spontaneous regression in most cases, aggressive treatment for vulvar high-grade squamous intraepithelial lesion is encouraged ${ }^{(17)}$.

A 75-year-old woman was referred to our unit for biopsy. Macroscopic examination revealed aceto-white changes on the left labia minora (Fig. 10). HFUS showed a nodular mass lesion arising from thickened epidermal entry echo. A sub-epidermal hypoechoic band confirmed that the lesion is restricted to the epidermis (Fig. 11). A histological examination confirmed VIN 3 (high-grade squamous intraepithelial lesion, Fig. 12).

\section{Discussion}

The aim of the research was to assess the utility of highfrequency ultrasonography in preoperative assessment of pathological vulvar lesions and a correlation between these images and histological findings. HFUS equipped with a $48 \mathrm{MHz}$ probe is particularly useful to assess the margin, depth and size of the lesion in most $\operatorname{cases}^{(18)}$. There are few reports on HFUS skin imaging, and so far this is the first report focusing on HFUS imaging in vulvar pathologies ${ }^{(19,20)}$. However, biopsy is a gold standard for the diagnosis of skin pathologies like tumors or VD that are resistant to standard

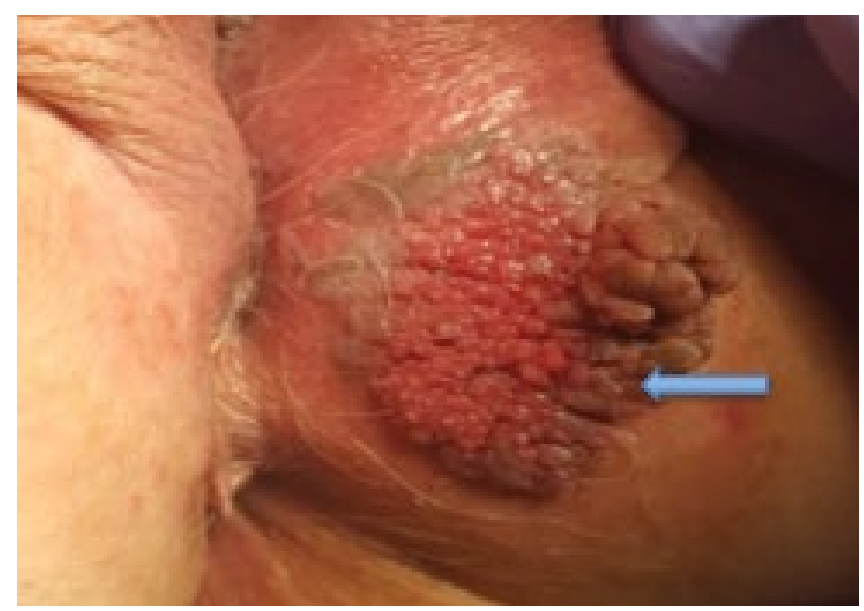

Fig. 4. Macroscopic image of condylomata acuminatum, large pappilar structures (arrow)

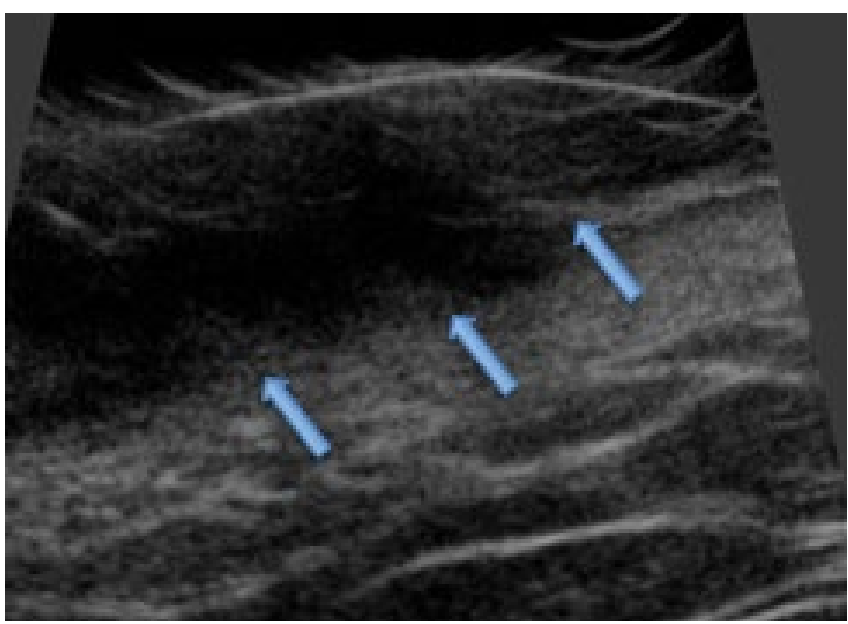

Fig. 5. HFUS image of condyloma acuminatum. Eminent hypoechoic lesion with sharp margin, well-differentiated from adjacent tissues (arrows). Subepidermal localisation

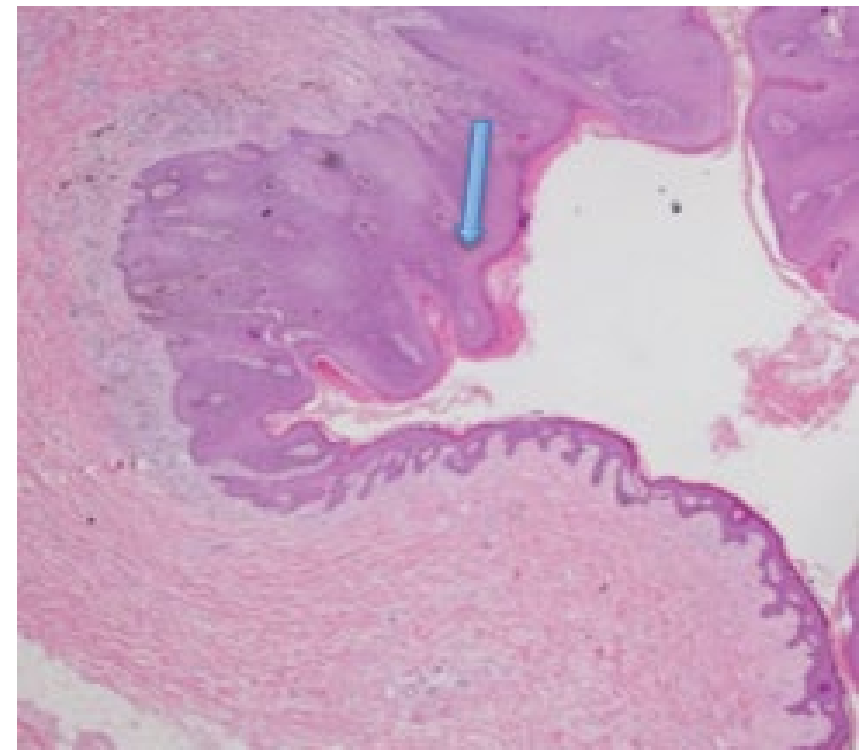

Fig. 6. Condylomata acuminatum histological image, hyperplastic dermal papillae with parakeratosis (arrow) ( $H$ and $E$ ) 


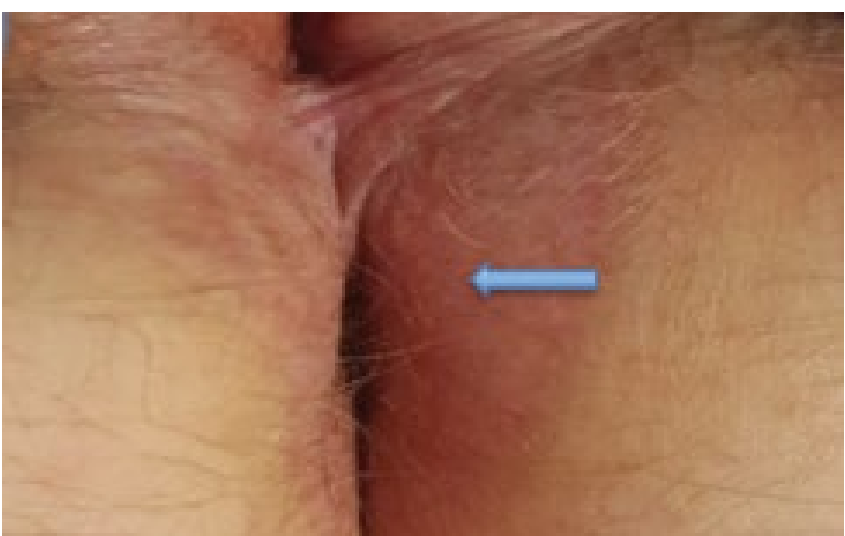

Fig. 7. Macroscopic image of Lichen sclerosus of vulva and anal region (arrow)

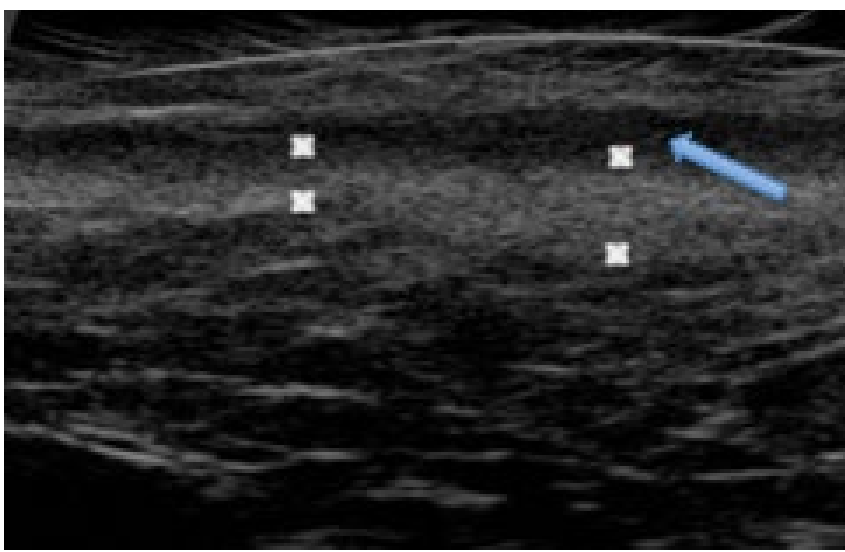

Fig. 8. Lichen sclerosus HFUS image. Hyperechoic entry echo with subepidermal low-echogenicity band (arrow). Thin dermis (between asterisks)

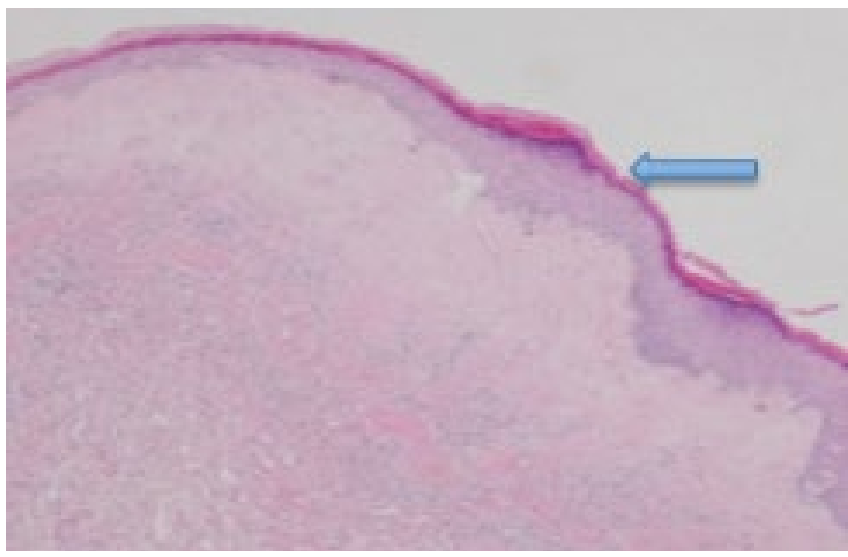

Fig. 9. Histological image of lichen sclerosus. Hyperkeratosis of dermis and epidermal atrophy (arrow) (H and $E)$

therapy. There is a define need for objective, non-invasive modality for diagnosis and localization of vulvar pathologies. HFUS serves this purpose as it may provide reasonably accurate information on vulvar skin pathologies, such as size, shape, depth and consistency before invasive skin biopsy or surgery ${ }^{(19)}$. These reports correspond with our observations. We were able to precisely assess vulvar pathologies,

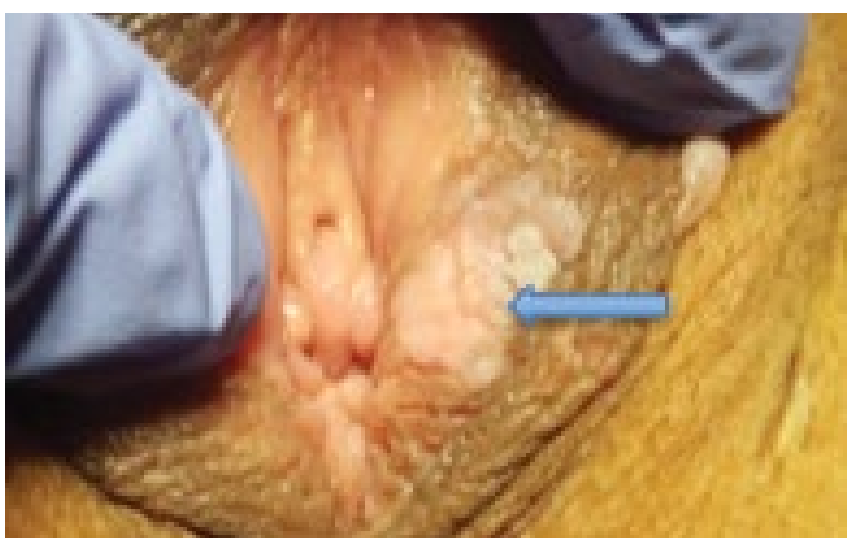

Fig. 10. Vulvar intraepithelial neoplasia 3 (VIN) - macroscopic image (arrow)

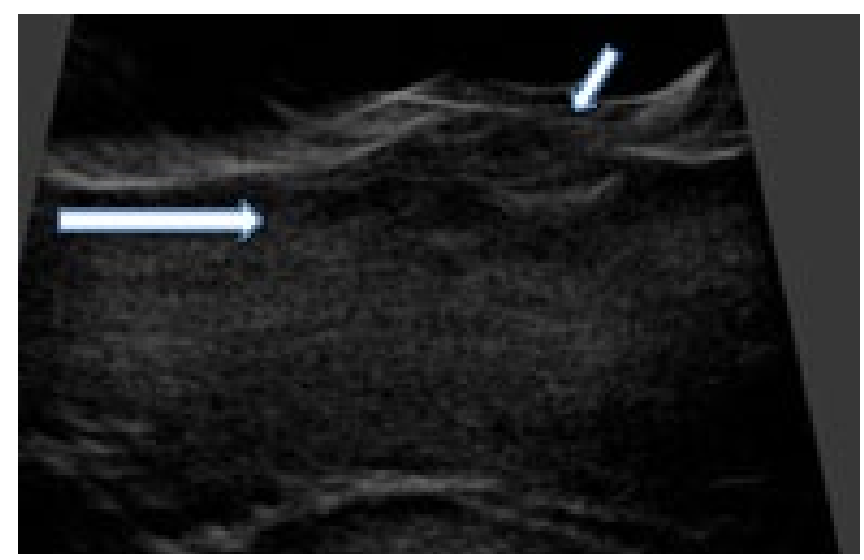

Fig. 11. HFUS image of VIN. Eminent lesion above epidermis (short arrow) with low-echogenicity band below epidermis (long arrow)

acquiring necessary information for better planning of the surgical procedure in 4 cases. Furthermore, in the case of a large condyloma, HFUS accurately showed the depth of the lesion, which was located within the dermis without deeper invasion to the subcutaneous tissue. However, is should be emphasized that ultrasound images depend on tissue composition, which influences the properties of the reflected sound wave. The greater the difference in acoustic impedance, the greater the reflection of the wave. In the case of folliculitis, LS and VIN, it was possible to see the echogenicity of the lesion. Condyloma was completely hypoechoic due to its dense structure. The echogenicity of the dermis is influenced by several factors, like orientation of collagen fibers, type of ground substances and water content ${ }^{(21-23)}$. In cases of folliculitis and LS, we noticed a subepidermal low-echogenicity band (SLEB). As it is not a pathognomic feature for these dermatoses, we would like to highlight the presence of this ultrasonographic finding. SLEB is present in many different conditions, such as inflammatory dermastoses (psoriasis, eczema, atopic dermatitis), mycosis fungoides, skin elastosis as well as in the photo-damaged skin ${ }^{(20-24)}$. To our knowledge, this is the first study to report VD imaging with HFUS. In clinical practice, however, in case of doubts in the differential diagnosis, histological evaluation of the skin biopsy specimen is the proceeding of choice. 


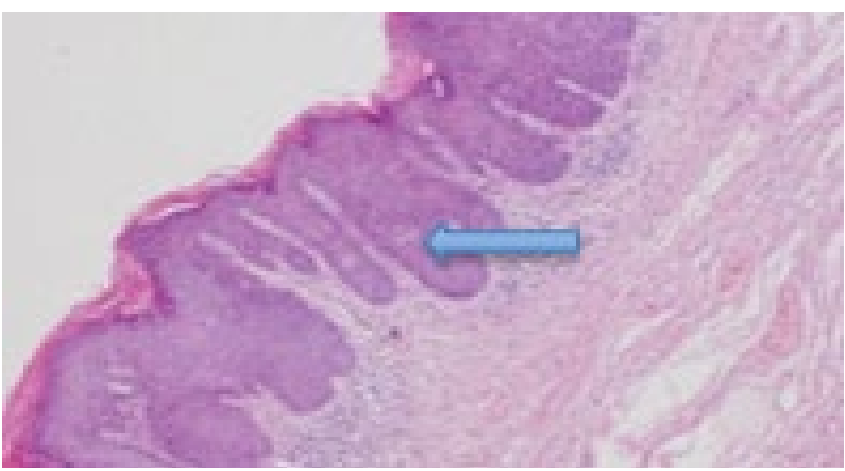

Fig. 12. Hisotological image of VIN. The full thickness of the epithelium is replaced with immature-appearing cells (arrow) (H and $E$ )

\section{References}

1. Sartori GC, Wicher RT, Ferreira FR, Batista VH: Most frequent dermatoses at a vulvar pathology outpatient clinic. An Bras Dermatol 2018; 93: 294-296.

2. Aarabi B, Mirvis S, Shanmuganathan K, Vaccaro AR, Holmes CJ, Akhtar-Danesh $\mathrm{N}$ et al.: Comparative effectiveness of surgical versus nonoperative management of unilateral, nondisplaced, subaxial cervical spine facet fractures without evidence of spinal cord injury: clinical article. J Neurosurg Spine 2014; 20: 270-277.

3. Barchino-Ortiz L, Suárez-Fernández R, Lázaro-Ochaita P: Vulvar inflammatory dermatoses. Actas Dermosifiliogr 2012; 103: 260-275.

4. Farage M, Maibach $\mathrm{H}$ : Lifetime changes in the vulva and vagina. Arch Gynecol Obstet 2006; 273: 195-202.

5. Stiles M, Redmer J, Paddock E, Schrager S: Gynecologic issues in geriatric women. J Womens Health (Larchmt) 2012; 21: 4-9.

6. Chan MP, Zimarowski MJ: Vulvar dermatoses: a histopathologic review and classification of 183 cases. J Cutan Pathol 2015; 42: 510-518.

7. Migda MS, Migda M, Migda B, Słapa RZ, Mlosek RK: Feasibility of using high-frequency skin ultrasound (HFSU) in vulvar skin assessment - initial report with the description of HFSU anatomy. Ginekol Pol 2016; 87: 19-25.

8. Mlosek RK, Malinowska S: Ultrasound image of the skin, apparatus and imaging basics. J Ultrason 2013; 13: 212-221.

9. Niazy F, Rostami K, Motabar AR: Giant condyloma acuminatum of vulva frustrating treatment challenge. World J Plast Surg 2015; 4: 159-162.

10. Stanley M: Immune responses to human papillomavirus. Vaccine 2006 24, Suppl 1: S16-22.

11. Hoang MP, Reutter J, Papalas JA, Edwards L, Selim MA: Vulvar inflammatory dermatoses: an update and review. Am J Dermatopathol 2014; 36: 689-704.

12. Simonetta C, Burns EK, Guo MA: Vulvar dermatoses: a review and update. Mo Med 2015; 112: 301-307.

13. Judson PL, Habermann EB, Baxter NN, Durham SB, Virnig BA: Trends in the incidence of invasive and in situ vulvar carcinoma. Obstet Gynecol 2006; 107: 1018-1022.

\section{Conclusion}

Our preliminary study confirms that HFUS is a useful tool in the assessment of vulvar pathologies, especially before surgical excision. A precise visualization of certain pathologies like folliculitis, LS, condylomata and VIN is possible. Further studies confirming our data are necessary.

\section{Conflict of interest}

Authors do not report any financial or personal connections with other persons or organizations, which might negatively affect the contents of this publication and/or claim authorship rights to this publication.

14. de Witte CJ, van de Sande AJ, van Beekhuizen HJ, Koeneman MM Kruse AJ, Gerestein CG: Imiquimod in cervical, vaginal and vulvar intraepithelial neoplasia: a review. Gynecol Oncol 2015; 139: 377-384.

15. Sideri M, Jones RW, Wilkinson EJ, Preti M, Heller DS, Scurry J et al.: Squamous vulvar intraepithelial neoplasia: 2004 modified terminology ISSVD Vulvar Oncology Subcommittee. J Reprod Med 2005; 50: 807-810.

16. van Seters M, van Beurden M, de Craen AJ: Is the assumed natural history of vulvar intraepithelial neoplasia III based on enough evidence? A systematic review of 3322 published patients. Gynecol Oncol 2005; 97: 645-651.

17. Wang L, Kawabe A, Kikugawa A, Takagi A, Kuromaki K: Vulvar intraepithelial neoplasia treated with a combination of surgical excision and laser ablation during pregnancy. Clin Case Rep 2018; 6: 1877-1879.

18. Mlosek RK, Słoboda K, Malinowska S: High frequency ultrasound imaging as a "potential” way of evaluation modality in side effects of lip augmentation - case report. J Cosmet Laser Ther 2019: 21: 203-205.

19. Bhatt KD, Tambe SA, Jerajani HR, Dhurat RS: Utility of high-frequency ultrasonography in the diagnosis of benign and malignant skin tumors. Indian J Dermatol Venereol Leprol 2017; 83: 162-182.

20. Polańska A, Dańczak-Pazdrowska A, Jałowska M, Żaba R, Adamski Z: Current applications of high-frequency ultrasonography in dermatology. Postepy Dermatol Alergol 2017; 34: 535-542.

21. Schmid-Wendtner MH, Dill-Müller D: Ultrasound technology in dermatology. Semin Cutan Med Surg 2008; 27: 44-51.

22. Jasaitiene D, Valiukeviciene S, Linkeviciute G, Raisutis R, Jasiuniene E Kazys R: Principles of high-frequency ultrasonography for investigation of skin pathology. J Eur Acad Dermatol Venereol 2011; 25: 375-382.

23. Dill-Müller D, Maschke J: Ultrasonography in dermatology. J Dtsch Dermatol Ges 2007; 5: 689-707.

24. Seidenari S: High-frequency sonography combined with image analysis: a noninvasive objective method for skin evaluation and description. Clin Dermatol 1995; 13: 349-359. 